EISSN: $2706-7947$ ISSN: 2077- 4613

DOI: $10.36632 /$ mejas/2021.11.3.52

Journal homepage: www.curresweb.com

Pages: 649-658

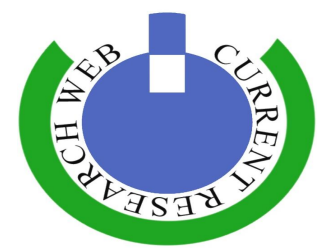

\title{
Role of Metformin and Estradiol in Alleviating the Risk Factors of Cardiovascular Dysfunction Induced by Ovariectomized Female Albino Rats
}

\author{
Nashwa M. Saied \\ Department of Hormone, National Organization for Drug Control and Research, Cairo, Egypt \\ Received: 08 July 2021 Accepted: 30 July $2021 \quad$ Published: 10 August 2021
}

\begin{abstract}
The current study used human equivalent therapeutic low dose of metformin to address its cardioprotective effect in ovariectomized (OVX) rats. Method: Forty adult female rats were equally divided into 4 groups. Group 1; sham operated group $(n=10)$, groups $2-4(n=30)$ were subjected to ovariectomy. After 1 month the groups 3, and 4 were assigned for the following treatments; Estradiol (E2) $(100 \mu \mathrm{g} / \mathrm{kg}$, i.m., every other day and Metformin (MF) $(100 \mathrm{mg} / \mathrm{kg} / \mathrm{day}$ orally. The study conducted for 1 month. Results: OVX-group showed a significant elevation in serum cardiac markers enzyme (CK-MB and LDH) where MF has superior effect on $\mathrm{E}_{2}$ in attaining their levels. Compared to OVX-group, MF-OVX and $\mathrm{E}_{2}-\mathrm{OVX}$ groups showed a significant decrease in serum Angiotensin II, cardiac MDA level and cardiac NO metabolite (NOx) with a significant increase in cardiac CAT activity and TAC. OVX-group showed a status of dyslipidemia while MF-OVX group showed normalized lipid profile markers, whereas the $\mathrm{E}_{2}-\mathrm{OVX}$ group showed a significant increase in the total cholesterol, and LDL-C levels. OVX -group showed a significant elevation in proinflammatory markers (TNF- $\alpha$ and IL-6). The treatments with MF and $\mathrm{E}_{2}$ were shown to significantly decrease the levels of both TNF- $\alpha$ and IL-6. Conclusion: By focusing on cardiac dysfunction markers, dyslipidemia and cardiac oxidative insult, it was found that the therapeutic dose of MF has a great merit in attenuating these markers over $\mathrm{E}_{2}$ treatment. Furthermore, MF has a similar effect as estradiol in mitigating inflammation status.
\end{abstract}

Keywords: metformin, cardiovascular dysfunction, Ovariectomy.

\section{Introduction}

Cardiovascular disease is a consequence of multiple metabolic disturbances and pathophysiological dysfunction. Dyslipidemia, which is a disturbance in lipid metabolism, leads to an elevation in LDL-C that consequently induced a lipid peroxidation and overproduction of ROS (Parthasarathy et al., 2010; Ito et al., 2019; Yamada et al., 2020). Several studies reported that the cardiovascular disease patients were associated with low concentration of antioxidant enzymes (Chrysohoou et al., 2007; Murr et al., 2009). The imbalance between the over production of free radicals (oxidant) and antioxidant leads to oxidative stress. The latter is implicated in many pathological dysfunctions such as activating the immune cell and enhances the production of inflammatory cytokines, inducing the endothelial dysfunction lading to myocardial infiltration with disturbing the permeability in cell membrane that leads to cardiac enzyme leakage. Furthermore, oxidative stress stimulates the renin-angiotensin system and elevates the level of angiotensin-II that leads to hypertension (Benigni et al., 2010; Mangge, 2014; Mittal et al., 2014).

Cardiovascular dysfunction (CVD) is a main cause of mortality and morbidity worldwide especially in a developing country, menopausal women are more prevalence to this (Roth et al., 2018; Zhu et al., 2019). Estradiol as a form hormonal replacement therapy is commonly used after menopause. It was documented that estradiol has antioxidant and anti-atherosclerotic activities and could attenuate the inflammation markers in ischemia/perfusion injury, also it may act as a short time

Corresponding Author: Nashwa M. Saied, Department of Hormone, National Organization for Drug Control and Research, Cairo, Egypt. E-mail: nashwasaeed1976@gmail.com 
vasodilator agent (Mendelsohn and Karas, 1999; Xu et al., 2006). However, the estradiol treatment may have undesirable adverse impact such as cancer (Brusselaers et al., 2018).

Metformin (MF) (insulin sensitizer) is an oral anti-hyperglycemic diguanide drug. It has been commonly used for the management of type two diabetes mellitus (T2DM), mostly in obese patients (Nasri and Rafieian-Kopaei, 2014). Several studies reported that high doses of MF are provided with favorable cardiovascular effects such as improving vascular dysfunction with reducing the vascular oxidative stress (Trott et al., 2011; Lobato et al., 2012; Oliveira et al., 2014). The maximum human therapeutic dose of MF is reported to be not exceeding $2550 \mathrm{mg} /$ day and the low dose is $500 \mathrm{mg}$ twice/ day (UK Prospective Diabetes Study (UKPDS) Group, 1998). According to allometric scaling used to safely extrapolate the MF dose for rats from human therapeutic dose, $100 \mathrm{mg} / \mathrm{kg} / \mathrm{day}$ metformin was used in the current study which equivalent to the daily low human therapeutic dose (Nair and Jacob, 2016).

From the reported studies, the present study aimed to assess the effect of low dose of Metformin (MF) based on human low therapeutic dose, on the cardiovascular disorders resulted from the surgical removal of ovaries (ovariectomized rats) in comparison with $17 \beta$-estradiol $\left(E_{2}\right)$ which is commonly used as hormonal replacement therapy.

\section{Materials and Methods}

\subsection{Chemicals}

$17 \beta$-estradiol $\left(\mathrm{E}_{2}\right)$ was purchased from Sedico Pharmaceutical Company; Giza, Egypt, $\mathrm{E}_{2}$ was dissolved in olive oil to prepare a dose of $100 \mu \mathrm{g} / \mathrm{kg}$. Metformin (MF) powder purchased from Sigma Aldrich Chemical Co. (St. Louis, MO, USA). MF was freshly prepared and dissolved in distilled water to prepare a dose of $100 \mathrm{mg} / \mathrm{kg}$. All other chemicals and reagents were of an analytical grade.

\subsection{Animals}

Forty adult Wister female albino rats weighing approximately 250-260 g, were obtained from the National Organization for Drug Control and Research. The animals were accommodated for two weeks before starting the experiment at $25 \pm 2^{\circ} \mathrm{C}$ and $55 \pm 5 \%$ humidity under a $12 \mathrm{~h}$ light/dark cycle and allowed free access to a standard diet and water ad labium. All experimental protocol were approved by Institutional Animal Ethics Committee at National Organization for Drug Control and Research (Approval no. 8/231/2019) and followed the Guide for the Care and Use of Laboratory Animals published by the U.S. National Institutes of Health (NIH Publication No. 85-23, revised 1996).

\subsection{Experimental design}

The animals were randomly allocated into four groups (10 rat per group) as follows: Group I, sham $(\mathrm{SH})$ operated, the remaining groups (2-4) were subjected to ovariectomy under aseptic conditions. Following 1 month of surgery the groups 1 and 2, received intramuscular injection of olive oil every other day. Groups 3 and 4 were assigned for the following treatments, Group $3\left(\mathrm{OVX}+\mathrm{E}_{2}\right)$ received intramuscular injection of $E_{2}$ dissolved in olive oil at a dose of $100 \mu \mathrm{g} / \mathrm{kg}$, every other day (Nomikos et al., 1987). Group 4, (OVX+ MF), MF received oral dose $100 \mathrm{mg} / \mathrm{kg} / \mathrm{day}$ (Wang et al., 2011). MF was freshly prepared and dissolved in distilled water. All treatments were conducted for one month.

The ovariectomy was carried out under aseptic conditions by removing the bilateral ovaries under mild intravenous anesthesia using $60 \mathrm{mg} / \mathrm{kg}$ thiopental (Lasota and Danowska-Klonowska, 2004). All treatment applications were carried out after one-month postoperative recovery.

\subsection{Preparation of cardiac homogenate for biochemical assays}

At the end of experiment, all groups were decapitated under mild anesthesia. Blood samples were collected from the retro-orbital vein of all rats into serum preparation tubes. Sera samples were divided into aliquots, stored at $-20{ }^{\circ} \mathrm{C}$ for further biochemical evaluation. Heart was immediately removed and washed in ice-cold saline, a number of ten hearts per group were weighed and 
homogenized in ice cold potassium chloride $(1.15 \% \mathrm{KCl})$ using a glass homogenizer to yield $10 \%$ homogenate for the determination of antioxidant parameters.

\subsection{Determination of serum estradiol level}

Serum rat estradiol was evaluated by ELISA kit purchased from BioSource co., Ltd., CA, USA.

\subsection{Determination of serum biochemical Risk factors of CVD}

Serum activities of lactate dehydrogenase enzyme (LDH) and creatine kinase MB (CK-MB) were assayed using commercial kits purchased from Reactivos GPL Barcelona, Spain. Serum TC, HDL-C and TG were determined using kits purchased from Biodiagnostic, Egypt. Low-density lipoprotein (LDL-C) was calculated according to the following equation; LDL-C = TC - HDL-C-TG/5 (Friedewald, Levy and Fredrickson, 1972). Atherogenic index 1 and atherogenic index 2 were calculated as TC/HDL-C and LDL-C/HDL-C ratios, respectively (Cai et al., 2017). Tumor necrosis factor- $\alpha(\mathrm{TNF}-\alpha)$, interleukin-6 (IL-6), and angiotensin-II (ANG-II) were evaluated using ELISA kits purchased from Cusabio, Germany.

\subsection{Determination of antioxidant/oxidant parameters in cardiac tissues}

Antioxidant and oxidant parameters were determined in cardiac tissue homogenate as follow: Catalase activity (CAT) was determined according to the method of Claiborne (1985), protein content for catalase activity was assayed according to the method of (Lowry et al.,1951), total antioxidant capacity (TAC) was evaluated using a commercial kit supplied by Bio-diagnostic, Cairo, Egypt. Malondialdehyde (MDA) was assayed according to the method of Mihara and Uchiyama (1978) and NO metabolite, nitrites and nitrates (NOx) was determined according to the method of Green et al.,(1982).

\subsection{Statistical analysis}

Data are recorded as means $\pm \mathrm{SD}$ ( $\mathrm{n}=8$ per group). The analysis of statistical significance was performed using one-way analysis of variance (ANOVA) followed by Tukey's multiple comparison test, $p<0.05$ was considered a significance. The data analyses were conducted using GraphPad Prism7 (GraphPad Software, San Diego CA, USA).

\section{Results}

\subsection{Effect of different treatments $\left(E_{2}\right.$ and $\left.M F\right)$ on serum estradiol $\left(E_{2}\right)$ level, serum $C K-M B$ and LDH enzyme activities \\ Table 1 revels that OVX-group showed a significant low level of serum $\mathrm{E}_{2}$ along with a high} significant increase in CK-MB and LDH activities, compared to $\mathrm{SH}$ group. OVX- $\mathrm{E}_{2}$ treated group showed a sudden increase in serum $\mathrm{E}_{2}$ level compared to OVX and $\mathrm{SH}$ groups, along with a significant reduction in CK-MB and LDH enzyme activities, compared to OVX group. Meanwhile, OVX-MF treated group showed a normalized level of serum estradiol in association with a significant reduction in CK-MB and LDH enzyme activities, compared to OVX group. The obtained results in the current study reveal the superior property of MF maintaining the estradiol level with improving the activities of myocardial enzymes levels than estradiol treatment.

Table 1: Effect of Estradiol and Metformin on the serum level of $17 \beta$ estradiol, CK-MB and LDH activity in ovariectomized rats

\begin{tabular}{llll}
\hline Treatment & $\mathbf{1 7} \boldsymbol{\beta}$ estradiol $(\mathbf{p g} / \mathbf{m l})$ & CK-MB $(\mathbf{U} / \mathbf{L})$ & LDH $(\mathbf{U} / \mathbf{L})$ \\
\hline SH & $88.14 \pm 4.80$ & $10.91 \pm 1.79$ & $200.56 \pm 80.58$ \\
OVX & $57.66 \pm 5.47^{\mathrm{a}^{* * *}}$ & $185.30 \pm 9.11^{\mathrm{a}^{* * *}}$ & $3070.69 \pm 692.14^{\mathrm{a}^{* * *}}$ \\
OVX+E & $131.60 \pm 5.19^{\mathrm{a}^{* * *} \mathrm{~b}^{* * *}}$ & $78.17 \pm 4.52^{\mathrm{a}^{* * *} \mathrm{~b}^{* * *}}$ & $683.99 \pm 141.57^{\mathrm{a}^{* * *} \mathrm{~b}^{* * *}}$ \\
OVX+MF & $81.22 \pm 6.54^{\mathrm{b}^{* * *} \mathrm{c}^{* * *}}$ & $30.61 \pm 5.40^{\mathrm{a}^{* * *} \mathrm{~b}^{* * *} \mathrm{c}^{* * *}}$ & $463.68 \pm 102.47^{\mathrm{b}^{* * *}}$ \\
\hline
\end{tabular}

The data are presented as the mean $\pm \mathrm{SD}, \mathrm{n}=8$, ${ }^{\mathbf{a}}$ significant difference versus the sham (SH) group; ${ }^{\mathbf{b}}$ significant difference versus the ovariectomized (OVX) group and ${ }^{\mathbf{c}}$ significant difference versus estradiol treatment. ${ }^{*} \mathrm{p}<0.05$, $* * \mathrm{p}<0.01$, and $^{* * *} \mathrm{p}<0.001$ 


\subsection{Effect of different treatments $\left(E_{2}\right.$ and $\left.M F\right)$ on cardiac antioxidant / oxidative parameter and} serum ANG-II level

Table (2) reveals that OVX group showed a significant elevation in cardiac MDA and serum ANG-II levels, along with a significant depletion in cardiac CAT activity and TAC, compared to SH group. OVX-E $E_{2}$ and OVX-MF treated groups showed normalized CAT enzyme activity in association with a significant decrease in ANG-II levels compared to OVX group. Both $\mathrm{E}_{2}$-and MF treatments induced a significant decline in cardiac MDA levels to be near the SH group. For cardiac TAC, it has been significantly elevated in OVX-MF treated group when compared to $\mathrm{SH}$, OVX, and OVX-E 2 treated group.

Table 2: Effect of Estradiol and Metformin on serum level of ANG-II, cardiac MDA, CAT activity and TAC in ovariectomized rats

\begin{tabular}{|c|c|c|c|c|}
\hline Treatment & ANG-II (pg./ml) & MDA ( $\mu \mathrm{mol} / \mathrm{g}$ tissue $)$ & CAT (U/mg protein) & TAC (mmol/L) \\
\hline SH & $8.75 \pm 0.78$ & $70.83 \pm 5.40$ & $0.23 \pm 0.04$ & $0.87 \pm 0.18$ \\
\hline OVX & $64.91 \pm 6.45^{\mathrm{a}^{* * *}}$ & $97.92 \pm 15.94^{\mathbf{a}^{* * * *}}$ & $0.04 \pm 0.01^{\mathrm{a}^{* * *}}$ & $0.48 \pm 0.14^{\mathbf{a}^{* * * *}}$ \\
\hline $\mathbf{O V X}+\mathbf{E}_{2}$ & $21.88 \pm 2.19^{\mathbf{a}^{* * * *} \mathbf{b}^{* * *}}$ & $75.39 \pm 10.19^{b^{* * *}}$ & $0.20 \pm 0.03^{\mathbf{a}^{*} \mathbf{b}^{* * * *}}$ & $0.90 \pm 0.11^{\mathbf{b}^{* * *}}$ \\
\hline OVX+MF & $25.95 \pm 4.15^{\mathbf{a}^{* * * *} \mathbf{b}^{* * * *}}$ & $72.33 \pm 8.69^{\mathbf{b}^{* * * *} \mathbf{c}^{* * * *}}$ & $0.23 \pm 0.04^{\mathbf{b}^{* * *}}$ & $1.34 \pm 0.13^{\mathbf{a}^{* * * *} \mathbf{b}^{* * * *}} \mathbf{c}^{* * * *}$ \\
\hline
\end{tabular}

The data are presented as the mean $\pm \mathrm{SD}, \mathrm{n}=8,{ }^{\mathbf{a}}$ significant difference versus the sham (SH) group; ${ }^{\mathbf{b}}$ significant difference versus the ovariectomized (OVX) group and ${ }^{\mathbf{c}}$ significant difference versus estradiol treatment. ${ }^{*} \mathrm{p}<0.05$, $* * \mathrm{p}<0.01$, and $^{* * *} \mathrm{p}<0.001$

\subsection{Effect of different treatments $\left(E_{2}\right.$ and $\left.M F\right)$ on serum lipid profile and atherogenic indices (AI-I and AI-II)}

Compared to SH group, ovariectomy induced a state of dyslipidemia evidenced by a significant increase in serum TC, TG, LDL-C levels with significant depletion of HDL-C level. Furthermore, it exhibited a significant elevation in atherogenic indices I and II (Table 3). $\mathrm{E}_{2}$ treatment induced a significant elevations in TC, and LDL-C compared to OVX- group. The recoded data revealed that MF treatment were more effective than $\mathrm{E}_{2}$ in improving the obtained state of dyslipidemia, as indicated by the attainment of significant improvement in lipid profile parameters and atherogenic indices.

Table 3: Effect of Estradiol and Metformin on the serum lipid profile levels of (TC), (TG), (HDL-C), (LDL-C) and atherogenic indices (AI-I and AI-II) in ovariectomized rats

\begin{tabular}{|c|c|c|c|c|c|c|}
\hline Treatment & $\begin{array}{c}\mathrm{TC} \\
(\mathrm{mg} / \mathrm{dl})\end{array}$ & $\begin{array}{c}\text { TG } \\
(\mathrm{mg} / \mathrm{dl})\end{array}$ & $\begin{array}{l}\text { LDL-C } \\
\text { (mg/dl) }\end{array}$ & $\begin{array}{l}\text { HDL-C } \\
(\mathrm{mg} / \mathrm{dl})\end{array}$ & AI-I & AI-II \\
\hline \multirow{2}{*}{ SH } & $111.60 \pm$ & $156.13 \pm$ & $41.62 \pm$ & $47.94 \pm$ & $1.89 \pm$ & $1.63 \pm$ \\
\hline & 7.39 & 4.20 & 10.45 & 8.04 & 0.49 & 0.41 \\
\hline \multirow{2}{*}{ OVX } & $162.52 \pm$ & $228.66 \pm$ & $85.18 \pm$ & $19.81 \pm$ & $7.25 \pm$ & $7.97 \pm$ \\
\hline & $24.20^{\mathrm{a}^{* * *}}$ & $11.09^{\mathrm{a}^{* * * *}}$ & $7.33^{\mathrm{a}^{* * * *}}$ & $4.80^{\mathrm{a}^{* * *}}$ & $1.43^{\mathrm{a}^{* * *}}$ & $1.43^{\mathrm{a}^{* * *}}$ \\
\hline \multirow{2}{*}{$\mathbf{O V X}+\mathrm{E}_{2}$} & $189.04 \pm$ & $186.88 \pm$ & $119.46 \pm$ & $30.90 \pm$ & $4.46 \pm$ & $5.07 \pm$ \\
\hline & $21.88^{\mathrm{a}^{* * *} \mathrm{~b}^{*}}$ & $7.71^{\mathrm{a}^{* * *}} \mathrm{~b}^{* * *}$ & $15.47^{\mathrm{a}^{* * *} \mathrm{~b}^{* * *}}$ & $7.27^{\mathrm{a}^{* * * *} \mathrm{~b}^{*}}$ & $1.40^{\mathrm{a}^{* * *} \mathrm{~b}^{* * *}}$ & $1.27^{\mathrm{a}^{* * *} \mathrm{~b}^{* * *}}$ \\
\hline \multirow{2}{*}{$\mathbf{O V X}+\mathbf{M F}$} & $122.15 \pm$ & $87.87 \pm$ & $48.00 \pm$ & $62.55 \pm$ & $1.65 \pm$ & $1.31 \pm$ \\
\hline & $12.60^{\mathrm{b}^{* * *} \mathrm{c}^{* * *}}$ & $10.93^{\mathrm{a}^{* * *} \mathrm{~b}^{* * *} \mathrm{c}^{* * *}}$ & $13.28^{\mathrm{b}^{* * *} \mathrm{c}^{* * *}}$ & $13.11^{\mathrm{a}^{* * *} \mathrm{~b}^{* * * *} \mathrm{c}^{* * *}}$ & $0.34^{b * * *} \mathrm{c}^{\mathrm{*} * *}$ & $0.32^{\mathrm{b}^{* * *} \mathrm{c}^{* * *}}$ \\
\hline
\end{tabular}

The data are presented as the mean $\pm \mathrm{SD}, \mathrm{n}=8 .{ }^{\mathbf{a}}$ significant difference versus the sham (SH) group; ${ }^{\mathbf{b}}$ significant difference versus the ovariectomized (OVX) group and ${ }^{\mathbf{c}}$ significant difference versus estradiol treatment. ${ }^{*} \mathrm{p}<0.05$, $* * \mathrm{p}<0.01$, and $^{* * *} \mathrm{p}<0.001$

\subsection{Effect of different treatments ( $E_{2}$ and MF) on serum TNF- $\alpha$, IL-6 and cardiac (NOx) metabolites}

Figure 1 demonstrate that OVX-group showed a significant elevation in serum proinflammatory markers, TNF- $\alpha$ (89.56; $\mathrm{p}<0.001)$, IL-6 (81.23; $\mathrm{p}<0.001)$ and cardiac (NOx) metabolite (195.3; $\mathrm{p}<0.001)$, compared to SH-group $(21.39,18.59$ and 69.14, respectively). OVX-E 2 and OVXMF groups showed a significant low levels of inflammatory cytokines; serum TNF- $\alpha$ (43.35 and $38.22 ; \mathrm{p}<0.001)$, IL-6 (42.78 and 37.12; $\mathrm{p}<0.001)$, compared to OVX-group, along with normal level of cardiac total NOx (69.38 and 69.87; $\mathrm{p}>0.001$ ), compared to SH-group. 

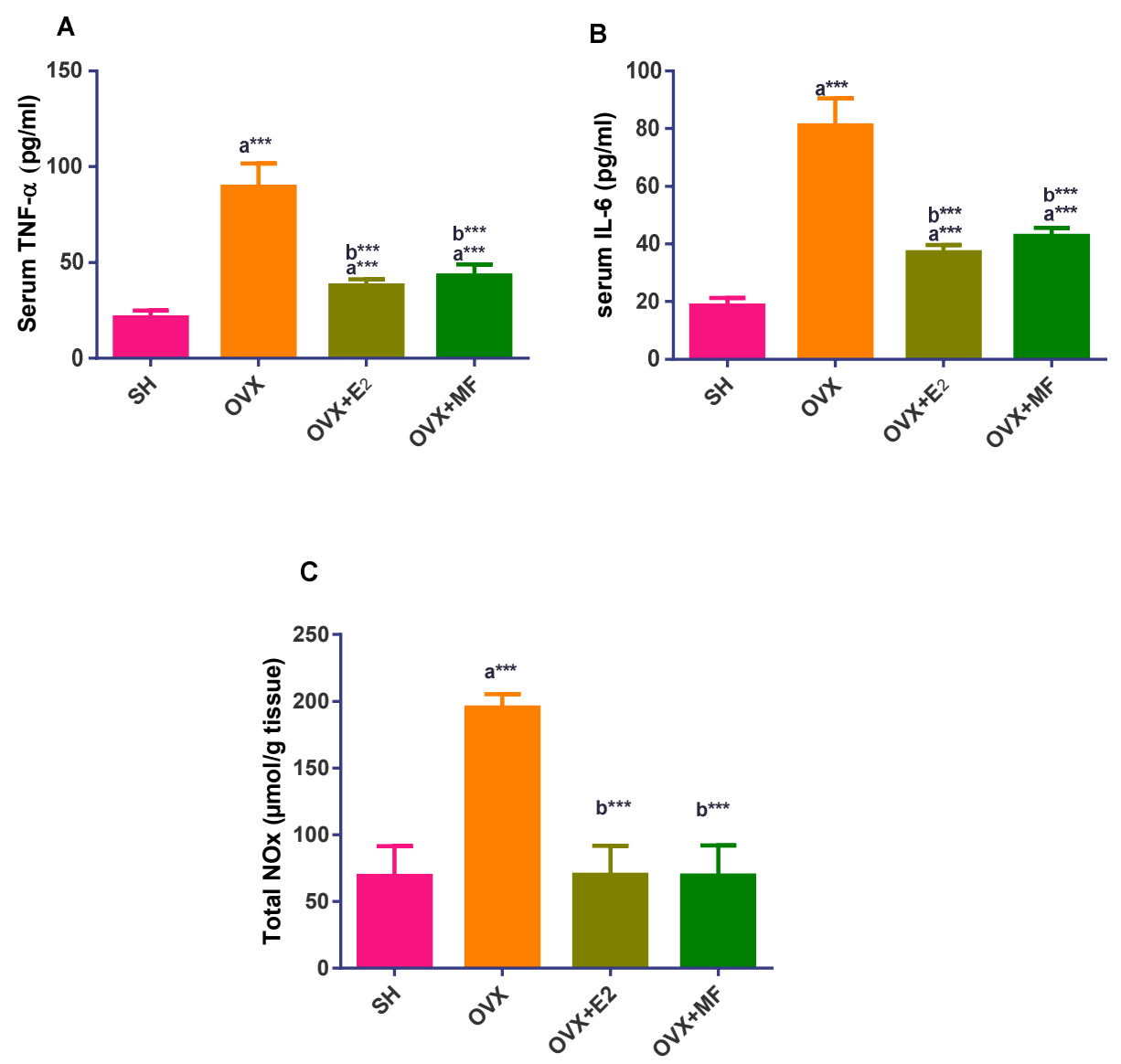

Fig. 1: Effect of estradiol $\left(E_{2}\right)$ and metformin $(M F)$ on $\mathbf{A}$ : tumor necrosis factor alpha (TNF $\left.\alpha\right)$, $\mathbf{B}$ : Interleukin 6 (IL-6) and $\mathbf{C}$ : Total nitrite and nitrate (NOx). The data are presented as the mean $\pm \mathrm{SD}, \mathrm{n}=8,{ }^{\mathbf{a}}$ significant difference versus the sham (SH) group; ${ }^{\mathbf{b}}$ significant difference versus the ovariectomized (OVX) group and ${ }^{\mathbf{c}}$ significant difference versus estradiol treatment $* \mathrm{p}<$ $0.05, * * \mathrm{p}<0.01$, and $^{* * *} \mathrm{p}<0.001$.

\section{Discussion}

It was documented that, deprivation of estrogen hormone activated the renin- angiotensin system (RAS) that leads to CVD (Yung et al., 2011). The results of the present study revealed that, ovariectomized rats suffered from a well- marked decrease in the serum level of $E_{2}$ that was associated with a significant increase in Angiotensin II (ANG II) which is a potent vasoconstrictor and a key factor in renin-angiotensin system (RAS) for maintaining the systemic arterial pressure (Manabe et al., 2005). It was documented that Ang II significantly stimulates the production of molecular oxygen species leading to mitochondrial dysfunction and cellular injury (de Cavanagh et al, 2007; Benigni et al., 2010).

The generation of oxidative stress in OVX rats is evidenced in the current study by the obtained increase in cardiac MDA level in concomitant with the reduction of catalase activity and association with a significant decrease in cardiac TAC level. This disturbance in redox status of cardiac tissue leads to dysfunction in membrane permeability leading to significant leakage of cardiac enzymes CK$\mathrm{MB}$ and LDH into the circulation that evidenced the induction of cardiac disorder.

Oxidative stress plays a crucial role in generating multiple pathophysiological process, it could increase the risk of CVD incidence such as atherosclerotic plaques and coronary artery disease (Stocker and Keaney, 2004; Rezzani et al., 2008; Kattoor et al., 2017). The obtained results of the present study revealed the incidence of a state of dyslipidemia throgh increasing in the atherogenic indices in OVX group. These findings are the harmony with those of (Phan and Toth, 2014; Cai et al., 2017; Wu et al., 2018; Ko and Kim, 2020) who reported that, dyslipidemia and serum atherogenic 
indices are higher in postmenopausal status. The increasing levels of cholesterol and low density lipoprotein especially LDL-C, which are vulnerable to the extensive oxidation by ROS, lead to lipid peroxidation products such as MDA product that accumulated in coronary arteries caused CVD. These findings could be attributed to the withdrawal of estradiol hormone in ovariectomized rats and proved the role of estrogen insufficiency in the production of oxidative insult in postmenopausal. This finding agrees with the results of (Parthasarathy et al., 2010; Ito et al., 2019; Yamada et al., 2020) who confirmed the role of estradiol withdrawal in the initiation and propagation of oxidative insults in menopause status.

Our finding revealed a significant elevation in pro-inflammatory cytokine TNF $\alpha$ and IL6 in OVX-treated group, which are considered a subcellular risk factors associated with CVD such as ischemia/perfusion, atherosclerosis and heart failure (Torre-Amione, 2005; Kaur et al., 2006). It was known that in heart and vasculature tissue, Ang II stimulates the inflammatory response through fostering the expression of pro-inflammatory chemokines, responsible for tissue accumulation of immunocompetent cells (Suzuki et al., 2003). Furthermore, this elevation in pro-inflammatory cytokines could be a result of elevated ROS generation that triggers the immune cells to migrate to the injured tissues as described by (Yang et al., 2007 and Mittal et al., 2014).

Focusing on the current results of NO production that was measured as NO metabolites, nitrites and nitrates (total NOx), ovariectomiezed rats displayed a significant increase in the level of NO metabolites, (NOx), compared to SH- group. NO plays a vasoprotective role and is constitutively expressed in endothelium by activation of eNOS. Under conditions of increased production of ROS in OVX group, eNOS becomes dysfunctional. Superoxide anions generated by the ROS systems combine with $\mathrm{NO}$ to form a highly reactive peroxynitrite $\left(\mathrm{OONO}^{-}\right)$and a reactive nitrogen species (RNS). That causes changes lead to endothelial dysfunction (Kuzkaya et al., 2003; Halliwell and Gutteridge, 2012). It combines with eNOS to form a reducing cofactors (such as tetrahydrobiopterin) [BH4]) and uncouples oxygen reduction from NO synthesis. Uncoupled eNOS produces superoxide anions instead of NO and hence is a ROS generator. (Kattoor et al., 2017). It has been reported that several factors induced up-regulation of NOS enzyme family such as a depletion in estrogen and an elevation of inflammatory markers, those factors are associated in OVX-group with concomitant

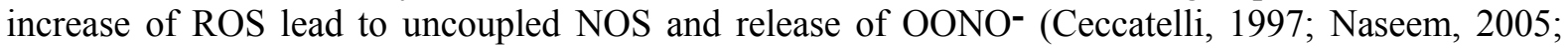
Ronchetti et al., 2016; Münzel et al., 2017).

This study shows that estrogen plays a functional role in protection against the cardiovascular disorder in ovariectomized rats through the modulation of angiotensin II, reduction of oxidative stress with improving the total antioxidant capacity and increasing the Catalase activity. Estradiol treatment reduced the elevation of pro-inflamatory cytokine and modulate the over production of (NOx), metabolite. This improving effects of estradiol replacement therapy is attributed to the antioxidant activity of estradiol that owing phenolic group on its structure which could scavenge the ROS species and maintain the normal oxidant status (Persky et al., 2000), Furthermore, it could upregulate the antioxidant enzyme expression (Suzuki et al., 2006).

Results of the current study elicited that ovariectomized rats treated with estradiol displayed a significant fluctuations in improving the disturbance of lipid profile. However the estradiol treatment caused a significant decrease in TG with increasing HDL-C levels and moderated the atherogenic indices when compared to OVX group, it caused a significant increase in cholesterol and LDL-C levels. This finding could be attribute to impaired in the hepatic steps of reverse cholesterol transport (RCT) this process can remove the cholesterol from peripheral tissues terminating and delivery of cholesterol to the liver for conversion to bile acids, which are ultimately delivered into the feces (Rosenson et al., 2012). LDL fraction a major carrier of cholesterol-binds about $60 \%$ of the total serum cholesterol. Although the function of LDL is to transport cholesterol to the tissues, LDL-C forms a part of the plasma membrane or can be converted into various metabolites, including steroid hormones (Sareen et al., 2005). Therefore, the function of LDL is not restricted to depositing cholesterol and other lipids in the peripheral cells. As LDL-targeted cells include cells of the vascular endothelium, high concentration and activity of LDL have serious implications in the etiology of CVDs (Cannon et al., 2019).

The pleotropic effect of metformin as antihyperlipidemic (Lin et al., 2018), antioxidant (DiazMorales et al., 2017), cardio-protective (Grant, 1996) with antidiabetic properties directed the current study to investigate its role in mitigating the risk factors of cardiovascular dysfunction induced by 
ovariectomy. Several studies were investigated the cardio-protective effect of high doses of MF ranging from 200 to $500 \mathrm{mg} / \mathrm{kg}$ on rats (Trott et al., 2011; Lobato et al., 2012; Oliveira et al., 2014). In the current study, low dose of MF was chosen simulated the daily human dose of metformin on the basis of its cardio-protective effect on adult male rats ( Yan et al., 2009; Wang et al., 2011; Nair and Jacob, 2016; Kheniser et al., 2019).

In our study, MF treatment normalized serum estradiol level in OVX-group, and attenuating ANG-II level, in concomitant with normalization of lipid profile this could be an important factors in diminishing the production of ROS, NOS and inflammation which evidenced by the reduction cardiac MDA, NOx, serum TNF $\alpha$ and IL-6 levels and augmenting cardiac CAT activity and TAC level in OVX-MF group. Furthermore, MF showed a substantial cardioprotective effect that emphasized by reduced the activity of CK-MB and LDH in the circulation. These finding are in the same line of those of (Alzoubi et al., 2014 and Zilinyi et al.,2018) who reported the efficacy of metformin in combatig the oxidative stress as well as its cardioprotective effect against doxorubicin-induced cardiotoxicity

Results of the current study confirmed the pleiotropic benefits of metformin attenuation of atherosclerosis (Forouzandeh et al., 2014), through restoring the serum levels of TC and LDL-C to be near the control value with decreasing TG and raising the HDL-C. Several studies attributed the potential hypolipidemic effect of metformin to increase $5^{-}$adenosine mono-phosphate AMP-activated protein kinase (AMPK) activity in rat and human hepatocytes also in human skeletal muscle in vitro (Zhou et al., 2001; Musi et al., 2002; Zhang et al., 2018) . AMPK is a serine-threonine kinase that in the liver inhibits the production of glucose, cholesterol and triglyceride and stimulates fatty acid oxidation through the regulation of several key proteins involved in lipid metabolism. (Velasco et al., 1997; Tokubuchi et al., 2017).

\section{Conclusion}

The pleotropic properties of MF as antioxidant, and antihyperlipidemic agent enable this drug to ameliorate the biochemical risk factors of cardiovascular dysfunction and inflammation induced following estradiol withdrew, which could be a safer and effective agent replaced the treatment with estradiol as a hormonal replacement therapy.

\section{Declarations}

Funding: Not applicable

Conflicts of interest: Not applicable

Availability of data and material: all raw data are applicable if needed

Code availability: Not applicable

\section{References}

Alzoubi, K., O. Khabour, S. Al-azzam, M. Tashtoush, and N. Mhaidat, 2014. Metformin Eased Cognitive Impairment Induced by Chronic L-methionine Administration: Potential Role of Oxidative Stress. Current Neuropharmacology, 12(2): 186-192.

Benigni, A., P. Cassis, and G. Remuzzi, 2010. 'Angiotensin II revixsited: New roles in inflammation, immunology and aging. EMBO Molecular Medicine., 2 (7): 247-257.

Brusselaers, N., R.M. Tamimi, P. Konings, B. Rosner, H.O. Adami, and J. Lagergren, 2018. 'Different menopausal hormone regimens and risk of Breast cancer. Annals of Oncology. 29(8): 1771-1776.

Cai, G., G. Shi, S. Xue, and W. Lu, 2017. 'The atherogenic index of plasma is a strong and independent predictor for coronary artery disease in the Chinese Han population. Medicine. Wolters Kluwer Health, 96(37): e8058.

Cannon, C.P., J.A. de Lemos, R.S. Rosenson, C.M. Ballantyne, Y. Liu, et al., 2019. Getting to an ImprOved Understanding of Low-Density Lipoprotein-Cholesterol and Dyslipidemia Management (GOULD): Methods and baseline data of a registry of high cardiovascular risk patients in the United States. Am. Heart J. 2019, 219, 70-77.

Ceccatelli, S.,1997. 'Expression and plasticity of NO synthase in the neuroendocrine system. Brain Research Bulletin., 44(4): 533-538. 
Chrysohoou, C., D.B. Panagiotakos, C. Pitsavos, J. Skoumas, M. Economou, L. Papadimitriou, and C. Stefanadis, 2007. 'The association between pre-hypertension status and oxidative stress markers related to atherosclerotic disease. Atherosclerosis., 192(1):169-176.

Claiborne, A., 1985. 'Catalase activity In: Greenwald RA (ed) Handbook of methods for oxygen free radical research. in. CRC Press,Boca Raton,Florida, 283-284.

Diaz-Morales, N., S. Rovira-Llopis, C. Bañuls, S. Lopez-Domenech, I. Escribano-Lopez, et al., 2017. 'Does Metformin Protect Diabetic Patients from Oxidative Stress and Leukocyte-Endothelium Interactions?. Antioxidants and Redox Signaling. 27(17): 1439-1445.

de Cavanagh, E.M., F. Inserra, M. Ferder, and L. Ferder, 2007. 'From mitochondria todisease: role of the renin-angiotensin system.. Am. J. Nephrol., 27: 545-553.

Forouzandeh, F., G. Salazar, N. Patrushev, S. Xiong, L. Hilenski, B. Fei, et al., 2014. Metformin beyond diabetes: pleiotropic benefits of metformin in attenuation of atherosclerosis. J Am Heart Assoc., 6 (3): e 001202.

Friedewald, W.T., R.I. Levy, and D.S. Fredrickson, 1972. Estimation of the concentration of lowdensity lipoprotein cholesterol in plasma, without use of the preparative ultracentrifuge.', Clinical chemistry, 18(6): 499-502.

Grant, P.J., 1996. The effects of high- and medium-dose metfformin therapy on cardiovascular risk factors in patients with type II diabetes. Diabetes Care, 19(1): 64-66.

Green, L.C., D.A. Wagner, J. Glogowski, P.L. Skipper, J.S. Wishnok, and S. R.Tannenbaum, 1982. 'Analysis of nitrate, nitrite, and [15N]nitrate in biological fluids. Analytical Biochemistry, 126(1):131-138.

Halliwell, B.I.M.C. and C.E.C. Gutteridge, 2012. Free radicals and antioxidants: Updating a personal View. Nutrition Review, 70: 257-265.

Ito, F., Y. Sono, and T. Ito, 2019. Measurement and clinical significance of lipid peroxidation as a biomarker of oxidative stress: Oxidative stress in diabetes, atherosclerosis, and chronic inflammation. Antioxidants. 8(3).

Kattoor, A.J., N.V.K. Pothineni, D. Palagiri, and J.L. Mehta, 2017. 'Oxidative Stress in Atherosclerosis. Current Atherosclerosis Reports, 19 (42): 1-11

Kaur, K., A.K. Sharma, S. Dhingra, and P.K. Singal, 2006. 'Interplay of TNF- $\alpha$ and IL-10 in regulating oxidative stress in isolated adult cardiac myocytes. Journal of Molecular and Cellular Cardiology, 41(6): 1023-1030.

Kheniser, K.G., S.R. Kashyap, and T. Kasumov, 2019. A systematic review: the appraisal of the effects of metformin on lipoprotein modification and function. Obesity Science and Practice., 1(5): $36-45$.

Ko, S.H. and H.S. Kim, 2020. Menopause-associated lipid metabolic disorders and foods beneficial for postmenopausal women. Nutrients, 1(12): 202-227

Kuzkaya, N., N. Weissmann, D.G. Harrison, and S. Dikalov, 2003. Interactions of peroxynitrite, tetrahydrobiopterin, ascorbic acid, and thiols: Implications for uncoupling endothelial nitricoxide synthase. Journal of Biological Chemistry, 278(25), pp. 22546-22554.

Lasota, A. and D. Danowska-Klonowska, 2004. 'Experimental osteoporosis--different methods of ovariectomy in female white rats. Roczniki Akademii Medycznej w Białymstoku (1995), 49 (1):129-131.

Lin, S.H., P.C. Cheng, S. Tu, Te, S.R. Hsu, Y.C. Cheng, and Y.H. Liu, 2018. 'Effect of metformin monotherapy on serum lipid profile in statin-naïve individuals with newly diagnosed type 2 diabetes mellitus, A cohort study',. PeerJ Inc., (6): e4578.

Lobato, N.S., F.P. Filgueira, G.N. Hagihara, E.H. Akamine, J.R. Pariz, R.C. Tostes, M.H.C. Carvalho, and Z.B. Fortes, 2012. 'Improveme. Life Sciences, 90(5-6): 228-235.

Lowry, O.H., N.J. Rosebrough, A.L. Farr, and R.J. Randall, 1951. Protein measurement with the folin. J. Biol. Chem., 193(1): 265-275.

Manabe, S., T. Okura, S. Watanabe, T. Fukuoka, and J. Higaki, 2005. 'Effects of Angiotensin II Receptor Block., Journal of Cardiovascular Pharmacology, 46(6): 735-739.

Mangge, H., 2014. 'Antioxidants, inflammation and cardiovascular disease. World Journal of Cardiology, 6(6): 462.

Mendelsohn, M.E. and R.H. Karas, 1999. 'The protective effects of estrogen on the cardiovascular system. New England Journal of Medicine, 340: 1801-1811. 
Mihara, M. and M. Uchiyama, 1978. 'Determination of malonaldehyde precursor in tissues by thiobarbituric acid test. Analytical Biochemistry, 86(1): 271-278.

Mittal, M., Siddiqui, M. R., Tran, K., S.P. Reddy, and A.B. Malik, 2014. 'Reactive oxygen species in inflammation and tissue injury. Antioxidants and Redox Signaling, 7 (20): 1126-1167.

Münzel, T., G.G. Camici, C. Maack, N.R. Bonetti, V. Fuster, and J.C. Kovacic, 2017. 'Impact of Oxidative Stress on the Heart and Vasculature: Part 2 of a 3-Part Series. Journal of the American College of Cardiology, 70 (2): 212-229.

Murr, C., B.M.Winklhofer-Roob, K. Schroecksnadel, Maritschnegg, et al., 2009. 'Inverse association between serum concentrations of neopterin and antioxidants in patients with and without angiographic coronary artery disease. Atherosclerosis, 202(2), pp. 543-549.

Musi, N., M.F. Hirshman, J. Nygren, M. Svanfeldt, P. Bavenholm, O. Rooyackers, et al., 2002. Metformin increases AMP-activated protein kinase activity in skeletal muscle of subjects with type 2 diabetes. Diabetes, 51(7): 2074- 81.

Nair, A.B. and S. Jacob, 2016. 'A simple practice guide for dose conversion between animals and human. Journal of basic and clinical pharmacy. 7(2): 27-31.

Naseem, K.M., 2005. 'The role of nitric oxide in cardiovascular diseases. Molecular Aspects of Medicine, 26(1-2): 33-65.

Nasri, H. and M. Rafieian-Kopaei, 2014. 'Metformin: Current knowledge. Journal of Research in Medical Sciences, 19 (7): 658-664.

Nomikos, G., C. Spyraki, A. Kazandjian, and A. Sfikakis, 1987. 'Estrogen treatment to ovariectomized rats modifies morphine-induced behavior. Pharmacology, Biochemistry and Behavior. 27(4): 611-617.

Oliveira, P.W.C., G.J. de Sousa, I.F. Caliman, A.Z. Lamas, A.R. Santos de Medeiros, T.U. de Andrade, G.R. de Abreu, S.G. de Figueiredo, and N.S. Bissoli, 2014. 'Metformin ameliorates ovariectomy-induced vascular dysfunction in non-diabetic wistar rats. Clinical Science. 127(4): $265-275$.

Parthasarathy, S., A. Raghavamenon, M.O. Garelnabi, and N. Santanam, 2010. 'Oxidized low-density lipoprotein. Methods in Molecular Biology, 610: 403-17.

Persky, A.M., P.S. Green, L. Stubley, C.O. Howell, L. Zaulyanov, G.A. Brazeau, and J.W. Simpkins, 2000. 'Protective effect of estrogens against oxidative damage to heart and skeletal muscle in vivo and in vitro. Proceedings of the Society for Experimental Biology and Medicine. 223(1): 5966.

Phan, B.A.P. and P.P. Toth, 2014. 'Dyslipidemia in women: Etiology and management. International Journal of Women's Health, 6: 185-194.

Rezzani, R., F. Bonomini, S. Tengattini, A. Fabiano, and R. Bianchi, 2008. 'Atherosclerosis and oxidative stress. Histology and Histopathology, 23(3): 381-390.

Ronchetti, S.A., L.I. Machiavelli, F.A. Quinteros, B.H. Duvilanski, and J.P. Cabilla, 2016. 'Nitric Oxide Plays a Key Role in Ovariectomy-Induced Apoptosis in Anterior Pituitary: Interplay between Nitric Oxide Pathway and Estrogen. PLOS ONE. 11(9): e0162455.

Rosenson, R.S., Jr.H.B. Brewer, W.S. Davidson, Z.A. Fayad, V. Fuster, J. Goldstein, et al., 2012. Cholesterol efflux and atheroprotection: advancing the concept of reverse cholesterol transport. Circulation, 125:1905-1919

Roth, G.A., D. Abate, K.H. Abate, S.M. Abay, et al., 2018. 'Global, regional, and national age-sexspecific mortality for 282 causes of death in 195 countries and territories, 1980-2017: a systematic analysis for the Global Burden of Disease Study 2017. The Lancet. 392(10159): $1736-1788$.

Sareen, S., J.L. Gropper, S., James, and L. Gro, 2005. In Advanced Nutrition and Human Metabolism, 4th ed., Gropper, S.S., Smith, J.L., Gro, J.L., Eds., Wadsworth, Inc.: Belmont, CA, USA, 159161.

Stocker, R. and J.F. Keaney, 2004. 'Role of oxidative modifications in atherosclerosis. Physiological Reviews, 84 (4): 1381-1478.

Suzuki, S., C.M. Brown, and P.M. Wise, 2006. 'Mechanisms of neuroprotection by estrogen. Endocrine, 29 (2): 209-215. 
Suzuki, Y., M. Ruiz-Ortega, O. Lorenzo, M. Ruperez, V. Esteban, and J. Egido, 2003. 'Inflammation and angiotensin II'. Int. J. Biochem. Cell Biol., 35: 881-900.

Tokubuchi, I., Y. Tajiri, S. Iwata, K. Hara, N. Wada, T. Hashinaga, et al., 2017. Beneficial effects of metformin on energy metabolism and visceral fat volume through a possible mechanism of fatty acid oxidation in human subjects and rats. PLoS ONE 12 (2): e0171293.

Torre-Amione, G., 2005. 'Immune activation in chronic heart failure. the American Journal of Cardiology., 11 (95): 3-8.

Trott, D.W., J.W. Seawright, M.J. Luttrell, and C.R. Woodman, 2011. 'NAD(P)H oxidase-derived reactive oxygen species contribute to age-related impairments of endothelium-dependent dilation in rat soleus feed arteries. Journal of Applied Physiology. 110 (5): 1171-1180.

UK Prospective Diabetes Study (UKPDS) Group, 1998. 'Effect of intensive blood-glucose control with metformin on complications in overweight patients with type 2 diabetes (UKPDS 34). Lancet. 352(9131): 854-865. doi: 10.1016/S0140-6736(98)07037-8.

Velasco, G., M.J. Geelen, and M. Guzman, 1997. Control of hepatic fatty acid oxidation by 5'-AMPactivated protein kinase involves a malonyl-CoA-dependent and a malonyl-CoA-independent

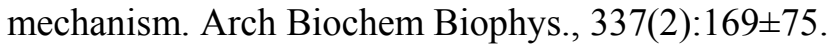

Wang, X.F., J.Y. Zhang, L. Li, X.Y. Zhao, H.L. Tao, and L. Zhang, 2011. 'Metformin improves cardiac function in rats via activation of AMP-activated protein kinase. Clinical and Experimental Pharmacology and Physiology, 38 (2): 94-101.

Wu, T.-T., Y. Gao, Y.Y. Zheng, Y.T. Ma, and X. Xie, 2018. Atherogenic index of plasma (AIP): a novel predictive indicator for the coronary artery disease in postmenopausal women. Lipids in Health and Disease, 17(1): 197.

Xu, Y., I.A. Arenas, S.J. Armstrong, W.C. Plahta, H. Xu, and S.T. Davidge, 2006. 'Estrogen improves cardiac recovery after ischemia/reperfusion by decreasing tumor necrosis factor- $\alpha$. Cardiovascular Research. 69(4): 836-844.

Yamada, T., K. Ogawa, T.D. Tanaka, T. Nagoshi, K. Minai, T. Ogawa, M. Kawai, and M. Yoshimura, 2020. 'Increase in oxidized low-density lipoprotein level according to hyperglycemia in patients with cardiovascular disease: A study by structure equation modeling. Diabetes Research and Clinical Practice, 161: 108036.

Yan, L., B. Kang, G. Li, Z.Yin, and Y. Wang, 2009. 'Effects of metformin on serum levels of sex hormone, leptin and insulin in ovariectomized Sprague-Dawley rats. Pharmazie - An International Journal of Pharmaceutical Sciences, 64 (12): 834-835.

Yang, D., S.G. Elner, Z.M. Bian, G.O. Till, H.R. Petty, and V.M. Elner, 2007. 'Pro-inflammatory cytokines increase reactive oxygen species through mitochondria and NADPH oxidase in cultured RPE cells. Experimental Eye Research, 85(4): 462-472.

Yung, L.M., W.T. Wong, X.Y. Tian, F.P. Leung, L.H. Yung, Z.Y. Chen, X. Yao, C.W. Lau, and Y. Huang, 2011. 'Inhibition of Renin-Angiotensin System Reverses Endothelial Dysfunction and Oxidative Stress in Estrogen Deficient Rats. PLoS ONE. 6(3): e17437.

Zhang, F., W. Sun, J. Chen, L. Jiang, P. Yang, Y. Huang, A. Gong, S. Liu, and S. Ma, 2018. 'SREBP2, a new target of metformin?. Drug Design, Development and Therapy, 12: 4163-4170.

Zhou, G., R. Myers, Y. Li, Y. Chen, X. Shen, J. Fenyk-Melody, et al., 2001. Role of AMP - activated Protein Kinase in mechanism of metformin action. The Journal of Clinical Investigation, 108 (8): 1167-1174

Zhu, D., H.F. Chung, A.J. Dobson, N. Pandeya, et al., 2019. 'Age at natural menopause and risk of incident cardiovascular disease: a pooled analysis of individual patient data, 4(11): e553-e564.

Zilinyi, R., A. Czompa, A. Czegledi, A. Gajtko, D. Pituk, I. Lekli, and A. Tosaki, 2018. 'The cardioprotective effect of metformin in doxorubicin-induced cardiotoxicity: The role of autophagy. Molecules, 23(5): 1184. 